\title{
The modification of short-term memory through instructions to forget ${ }^{1}$
}

\author{
ROBERT A. BJORK, UNIVERSITY OF MICHIGAN \\ DAVID LaBERGE AND ROSS LEGRAND, UNIVERSITY OF MINNESOTA
}

This study was designed to investigate whether Ss could reduce the proactive interference of a first verbal item $(\mathrm{CCCC})$ if they were signalled just prior to the presentation of a second item that they could forget the first item. Recall of the second of two CCCC items inserted in a series of digits was better when Ss were told to "drop" the first item than when there was no such instruction, but it was not as good as recall when only one item was presented.

To the degree that proactive interference (PI) is observed in short-term memory, it could derive from one or more of several sources. In the standard shortterm memory task, a $S$ is presented some number of verbal items and he is asked to output one or more of them after an elapsed retention interval. The early items could interfere with the recall of a given later item in several ways. (1) The item may be misperceived or mis-stored when it is presented because the $S$ is attending to (rehearsing) earlier items. (2) Rehearsal of the item during the retention interval may be impaired to the extent that the Suses the available rehearsal time to rehearse one or more of the earlier items. (3) At the time the item is to be recalled, the early items may provide competing responses. (4) Finally, the S may not exert maximum effort in recalling the item if subsequent recalls of earlier items are required.

One method of assessing the relative importance of these possible sources of PI is to eliminate some of them experimentally and examine the amount of PI remaining. Source (4) above can be eliminated by requiring that only one item be recalled and Source (1) can be minimized by avoiding short presentation times. Sources (2) and (3) are not so easy to eliminate and in standard procedures are perfectly confounded.

One attempt to vary the number of PI items while holding the memory load constant is described in a study by Bjork (1967). In this experiment Ss were presented lists of paired associates some of which contained a signal to forget all pairs presented prior to the signal because the tested (probed) pair would be one of those presented after the signal. Bjork found no effect on recall of the number of pairs presented prior to the forget signal; that is, there was no PI owing to the "forgotten" pairs.

The present study utilizes a "drop" instruction in a short-term memory situation that minimizes opportunities for more than one rehearsal per item. Two items are inserted in a series of digits that the $S$ is required to shadow, and the $S$ is sometimes told to drop the first item by a signal given just before the appearance of the second item.

\section{Method}

The Ss were 24 men and women volunteers from an introductory psychology course at the University of Minnesota. Every S was presented 48 lists on a Lafayette memory drum. Each list consisted of a series of digits (cf., LaBerge \& Winokur, 1965) plus one or two consonant quadragrams (CCCC items) inserted in the digit series. The digits were colored red or black in a random fashion, omitting runs greater than two of the same color or digit. In constructing the $\mathrm{CCCC}$ items, $\mathrm{Q}, \mathrm{W}$, $\mathrm{X}$, and $\mathrm{Z}$ were omitted and similarity of sounds within each item was minimized (Wickelgren, 1966).

The Ss were required to call out (i.e., shadow) the color and value of each digit. The CCCC items were always colored black and when one appeared in the series the Ss were required to read it aloud. Every $\mathrm{CCCC}$ item was preceded by asterisks to the right of the two digits just preceding the item to signal the $S$ that an item was about to appear. At the end of each list the word "item" appeared as a recall cue for one or both of the CCCC items.

For both digits and $\mathrm{CCCC}$ items the presentation rate was $1 \mathrm{sec}$, and consecutive lists were separated by $8 \mathrm{sec}$. Three horizontal warning lines preceded each list.

Condition 1. These lists contained two CCCC items separated by four or eight digits. The first item was preceded by two, four, or six digits and there were zero, four, eight, or twelve digits between the second item and the end of the list. In this condition the Ss were required to recall both items, second item first, at the end of the list.

Condition 2. These lists were identical to Condition 1 except that colored dots appeared to the left of the two digits just preceding the second $\mathrm{CCCC}$ item. The colored dots served as a signal to the $S$ that he could forget the first item because he would have to recall only the second item.

Condition 3. These were control lists in which there was only one CCCC item placed at positions corresponding to the positions of the second CCCC item in Conditions 1 and 2 .

The four retention intervals $(0,4,8$, or $12 \mathrm{sec})$ and the three conditions generated the 12 possible combinations. Each of the 12 combinations was given in a block of 12 lists, omitting runs greater than two of lists with the same retention interval or condition. The $\mathrm{CCCC}$ items could be removed from the memory drum tapes so that, across Ss, each item appeared in every condition and retention interval combination. Order of presentation of the blocks was counterbalanced and the 


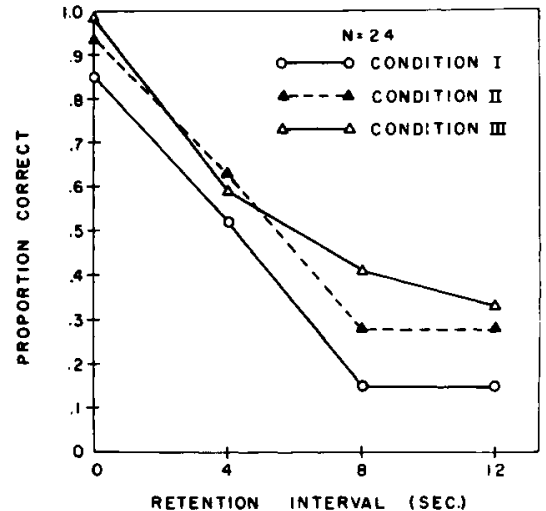

Fig. 1. Proportion of correct recalls. Condition 1: two items presented, recall both. Condition 2: two items presented, recall second only. Condition 3: only one item presented and recalled.

order of conditions and retention intervals was randomized within each block.

\section{Resulis}

Figure 1 shows, for each of the Conditions 1, 2, and 3 , the proportion of completely correct recalls (all four consonants recalled in the correct order) at each retention interval. Only the data from lists in which no shadowing errors occurred are included. Since the spacing of the two CCCC items had no apparent effect in Conditions 1 and 2, the data from the 4 sec and 8 sec spacings are combined in Fig. 1.

The differences in performance levels between the conditions at the longer retention intervals were tested by an analysis of variance. The data from each $\mathrm{S}$ at the $8 \mathrm{sec}$ and $12 \mathrm{sec}$ intervals were averaged and a two-way analysis of variance was applied to the averages followed by linear contrasts of pairs of conditions. The overall $F$ test was significant beyond the .01 level, the linear contrasts between Conditions 1 and 2 and 1 and 3 were significant at the .01 level, and the contrast between Conditions 2 and 3 was significant at the .05 level.

The obtained difference between Conditions 1 and 2 must be qualified by the fact that the Condition 1 curve lies well below the Condition 2 curve at the 0 sec retention interval. It is possible that under Condition 1 some Ss failed to store the second item when it was presented, so that the retention curve reflects some failure to "learn" as well as a decline in the retention of what was learned.

An effort was made to equate the conditions for initial learning by analyzing only the data from the subset of Ss who had perfect recall at the 0 sec retention interval under all conditions. Figure 2 displays the performance of the $12 \mathrm{Ss}$ who belonged to this subset. It should be noted that this analysis is valid only to the extent the subset of Ss defined on the basis of perfect responding at the 0 sec interval does not contain Ss who failed to store the second item in lists with longer retention intervals.

An analysis of variance and linear contrasts were performed as before on the data from the $8 \mathrm{sec}$ and 12 sec retention intervals. The overall $\mathrm{F}$ was highly signif-

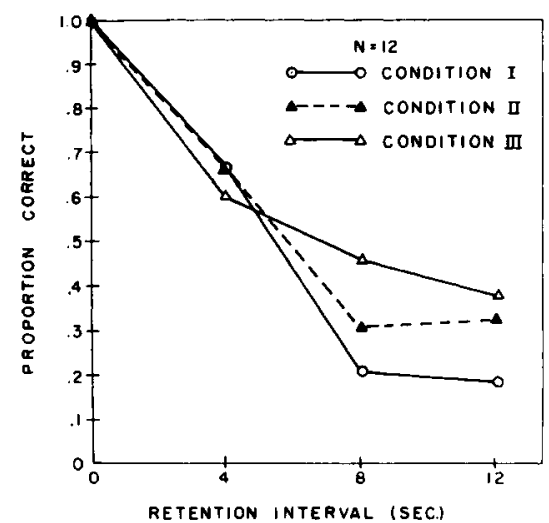

Fig. 2. Proportion of correct recalls for the subset of Ss who made no errors at the 0-sec retention interval.

icant, the linear contrasts between Conditions 1 and 3 and 1 and 2 were significant at the .01 and .05 levels, respectively, but the contrast between Conditions 2 and 3 did not attain significance. Thus, the pattern of results shown in Fig. 1 holds up in Fig. 2, with the indication that performance under Condition 2 is closer to performance under Condition 3 than it is to performance under Condition 1.

\section{Discussion}

The clear superiority of performance under Condition 3 compared to that under Condition 1 indicates that when the $S$ is required to recall both items, the first item produces considerable proactive interference on the recall of the second.

The fact that the "drop" instruction of Condition 2 results in a performance level between those of Conditions 1 and 3 can be interpreted in several ways. (1) It is an intriguing but highly unlikely possibility that Ss can actually erase items from short-term memory upon demand. (2) Relieving the $S$ of the responsibility of remembering the first item may allow him to rehearse the second item more effectively during the retention interval than is possible in Condition 1. This interpretation also seems unlikely since the experimental task was designed to virtually prohibit rehearsal. (3) In Condition 1 the demand that the first item be recalled after the second item is recalled could lead to a less efficient initial recall compared to Condition 2. (4) Ss may be able to respond to the drop instruction by actively tagging or coding one of the two items in a way that reduces the interference between them.

\section{References}

BJORK, R. A. The effect of instructions to selectively forget during short-term memory, Memorandum Rep. No. 3, Human Performance Center, University of Michigan, 1967.

LaBERGE, D., \& WINOKUR, S. Short-term memory using a visual shadowing procedure. Psychon. Sci., 1965, 3, 239-240.

WICKELGREN, W. Phonemic similarity and interference in short-term memory for single letters. J. exp. Psychol, 1966, 71, 396-404.

Note

1. This research was supported by the Center for Research in Human Learning at the University of Minnesota (NSF Grant GS-541). 